\title{
Elevated CENPW expression distinguishes basal-like human breast cancer.
}

Shahan Mamoor, MS ${ }^{1}$

$3 \quad$ shahanmamoor@gmail.com

East Islip, NY 11730

Patients diagnosed with basal-like breast cancer face a more aggressive disease course and more dismal prognosis than patients diagnosed with luminal A and luminal B breast cancer molecular subtypes (1-4). We mined published microarray data $(5,6)$ to understand in an unbiased fashion the most distinguishing transcriptional features of tumors from patients with basal or basal-like subtype breast cancer. We observed transcriptome-wide differential expression of centromere protein $\mathrm{W}$, CENPW, when comparing tumors of patients with basal-like breast cancer with that of other PAM50 molecular subtypes. CENPW mRNA was present at significantly higher quantities in the tumors of patients with basal-like breast cancer. Analysis of patient survival data revealed that CENPW primary tumor expression was correlated appears to distinguish basal-like human breast cancer from the other molecular subtypes.

Keywords: basal subtype, basal-like breast cancer, CENPW, systems biology of breast cancer, targeted therapeutics in breast cancer, molecular subtype 
Classification of disease types and disease subtypes enables development of enhanced diagnostic capabilities, prognostic information, as well as an understanding of human disease that can support superior medical, surgical and radiological treatment of complex diseases such as cancer. Molecular subtypes in human breast cancer include luminal A, luminal B, basal or basal-like, and HER2+. Systems-level analyses, such as comparative tumor transcriptome analyses, can inform such approaches. We mined published microarray data $(5,6)$ to identify the most significant transcriptional differences when comparing human breast cancer molecular subtypes. Integrative microarray dataset analysis has provided data illuminating genes whose expression define basal-like subtype breast cancers, and we present evidence here.

\section{Methods}

We utilized datasets GSE74667 (5) and GSE87049 (6) for this global differential gene expression analysis of basal subtype human breast cancer in conjunction with GEO2R. GSE74667 was generated using Agilent-014850 Whole Human Genome Microarray 4x44K G4112F technology with $n=23$ basal-like breast tumors and $n=72$ breast tumors of other molecular subtypes (luminal A, luminal B, HER2-enriched, and normal-like); analysis was performed using platform GPL6480. GSE87049 was generated using Affymetrix Human Gene 1.0 ST Array [transcript (gene) version] technology with $n=14$ basal-like breast tumors and $n=109$ breast tumors of other molecular subtypes (luminal A, luminal B, HER2-enriched, and normal-like); analysis was performed using platform GPL6244. The Benjamini and Hochberg method of $p$-value adjustment was used for ranking of differential expression but raw $p$-values were used to assess statistical significance of global differential expression. Log-transformation of data was auto-detected, and the NCBI generated category of platform annotation was used. A statistical test was performed to evaluate whether CENPW gene expression was significantly different between the tumors of patients with basal and non-basal molecular subtype breast cancer using a two-tailed t-test with Welch's correction (PRISM 9.1.1 (225)). For Kaplan-Meier survival analysis, we used the Kaplan-Meier plotter tool (8) for correlation of CENPW mRNA expression levels with overall survival in $n=943$ breast cancer patients, $n=296$ basal subtype breast cancer patients, $n=222$ luminal A subtype breast cancer patients, $n=200$ luminal B subtype breast cancer patients, $n=198$ HER2+ subtype breast cancer patients, and $n=27$ normal-like subtype breast cancer patients.

\section{Results}

CENPW is among the genes whose expression is most different when comparing basal-like breast cancers with breast cancers of other molecular subtypes.

We used published microarray data (5) to compare the whole tumor transcriptomes of 23 patients with basal subtype breast cancer and 72 patients with breast cancer of other molecular subtypes (luminal A, luminal B, HER2+ and normal-like) to identify genes whose expression marked the basal-like molecular subtype. We observed transcriptome-wide differential expression of centromere protein W, CENPW, when comparing the tumors of patients with basal-like cancer with that of patients with luminal A, luminal B and HER2+ cancers (Table 1). When sorting each of the transcripts whose expression was measured globally, CENPW expression ranked 30 out of 31157 total transcripts, equating to $99.9 \%$ differential expression; this was statistically significant ( $p$-value: $1.68 \mathrm{E}-13)$.

Analysis of a second published microarray dataset, comparing tumor transcriptome data from a separate cohort of patients (14 patients with basal-like breast cancer and 109 patients with breast cancer of other molecular subtype (luminal A, luminal B, HER2+ and normal-like) (5) revealed transcriptome-wide differential expression of CENPW in patients with basal subtype human breast cancer (Table 2). When sorting each of the transcripts whose expression was measured globally, CENPW expression ranked 55 out 
of 33297 total transcripts, equating to $99.8 \%$ differential expression; this was statistically significant ( $p$-value: 4.13E-17). Thus, the data suggested differential expression of CENPW was a fundamental feature of basal-like breast cancer in humans rather than an artifact of a single microarray dataset or

$\underline{\text { CENPW expression is significantly higher in basal-like breast cancers. }}$

We obtained exact CENPW mRNA expression data for basal-like breast tumors and tumors from other molecular subtypes (luminal A, luminal B, HER2+ and normal-like) to determine magnitude and direction of CENPW gene expression change in basal-like breast cancer. CENPW was expressed at higher levels in the tumors of patients with basal-like breast cancer as compared to the tumors of patients from other PAM50 molecular subtypes, and this difference was statistically significant (Figure $1 ; p<0.0001$ ). CENPW was expressed at $0.037 \pm 0.870$ arbitrary units (A.U.) in basal-like breast cancer and $-1.549 \pm$ 0.752 A.U. in all other molecular subtypes (Figure 1).

$\underline{\text { CENPW expression correlates with overall survival in basal-like and luminal A subtype breast cancer }}$ patients.

We asked whether primary tumor expression of CENPW was correlated with survival outcomes in patients with basal-like breast cancer. We observed a correlation between primary tumor expression of CENPW and overall survival (OS) in patients with basal-like breast cancer which was statistically significant (Figure 2; $p$-value: 0.042; hazard ratio: 0.61 (0.38-0.99)). CENPW primary tumor expression was a positive prognostic indicator in patients with basal-like breast cancer. Basal-like patients with low tumor expression of CENPW possessed, on average, markedly shorter OS as compared to basal-like patients with high tumor expression of CENPW (Table 3).

We also observed a correlation between primary tumor expression of CENPW and overall survival (OS) in patients with luminal A breast cancer which was statistically significant (Figure 2; $p$-value: 0.036; hazard ratio: 2.19 (1.03-4.56)). CENPW primary tumor expression was a negative prognostic indicator in patients with luminal A breast cancer. Luminal A patients with low tumor expression of CENPW possessed median OS of 169.2 months, while luminal A patients with high tumor expression of CENPW possessed median OS of 106.8 months (Table 3).

CENPW primary tumor expression was not correlated with overall survival in all patients (irrespective of molecular subtype) (Figure 2; $p$-value: 0.18; hazard ratio: 1.2 (0.92-1.57)), in luminal B subtype breast cancer (Figure 2; $p$-value: 0.81; hazard ratio: 1.07 (0.61-1.87), in HER2+ breast cancer (Figure 2; $p$-value: 0.37 ; hazard ratio: $0.78(0.45-1.34)$ ), or in normal-like breast cancer (Figure 2; $p$-value: 0.2 ; hazard ratio: $0.42(0.1-1.68))$.

\section{Discussion}

We find here that CENPW is among the genes whose expression is most different when comparing basal-like breast cancers (BLBC) to cancers of other molecular subtypes, with significantly more CENPW mRNA in BLBC. Kaplan-Meier analysis of patient survival data revealed that CENPW expression was correlated with overall survival in human patients with basal-like and luminal A breast cancer, with lower expression of CENPW associated with inferior outcomes. CENPW may be one factor that specifies tumor subtype identity in basal-like breast cancer. 


\section{References}

1. Rakha, E.A., Reis-Filho, J.S. and Ellis, I.O., 2008. Basal-like breast cancer: a critical review. Journal of clinical oncology, 26(15), pp.2568-2581.

2. Milioli, H.H., Tishchenko, I., Riveros, C., Berretta, R. and Moscato, P., 2017. Basal-like breast cancer: molecular profiles, clinical features and survival outcomes. BMC medical genomics, 10(1), pp.1-17.

3. Foulkes, W.D., Smith, I.E. and Reis-Filho, J.S., 2010. Triple-negative breast cancer. New England journal of medicine, 363(20), pp.1938-1948.

4. Dietze, E.C., Sistrunk, C., Miranda-Carboni, G., O'regan, R. and Seewaldt, V.L., 2015. Triple-negative breast cancer in African-American women: disparities versus biology. Nature Reviews Cancer, 15(4), pp.248-254.

5. She, Q.B., Gruvberger-Saal, S.K., Maurer, M., Chen, Y., Jumppanen, M., Su, T., Dendy, M., Lau, Y.K.I., Memeo, L., Horlings, H.M. and van de Vijver, M.J., 2016. Integrated molecular pathway analysis informs a synergistic combination therapy targeting PTEN/PI3K and EGFR pathways for basal-like breast cancer. BMC cancer, 16(1), pp.1-16.

6. Romero-Cordoba, S.L., Salido-Guadarrama, I., Rebollar-Vega, R., Bautista-Piña, V., Dominguez-Reyes, C., Tenorio-Torres, A., Villegas-Carlos, F., Fernández López, J.C., Uribe-Figueroa, L., Alfaro-Ruiz, L. and Hidalgo-Miranda, A., 2021. Comprehensive omic characterization of breast cancer in Mexican-Hispanic women. Nature communications, 12(1), pp.1-19.

7. Györffy, B., Lanczky, A., Eklund, A.C., Denkert, C., Budczies, J., Li, Q. and Szallasi, Z., 2010. An online survival analysis tool to rapidly assess the effect of 22,277 genes on breast cancer prognosis using microarray data of 1,809 patients. Breast cancer research and treatment, 123(3), pp.725-731. 


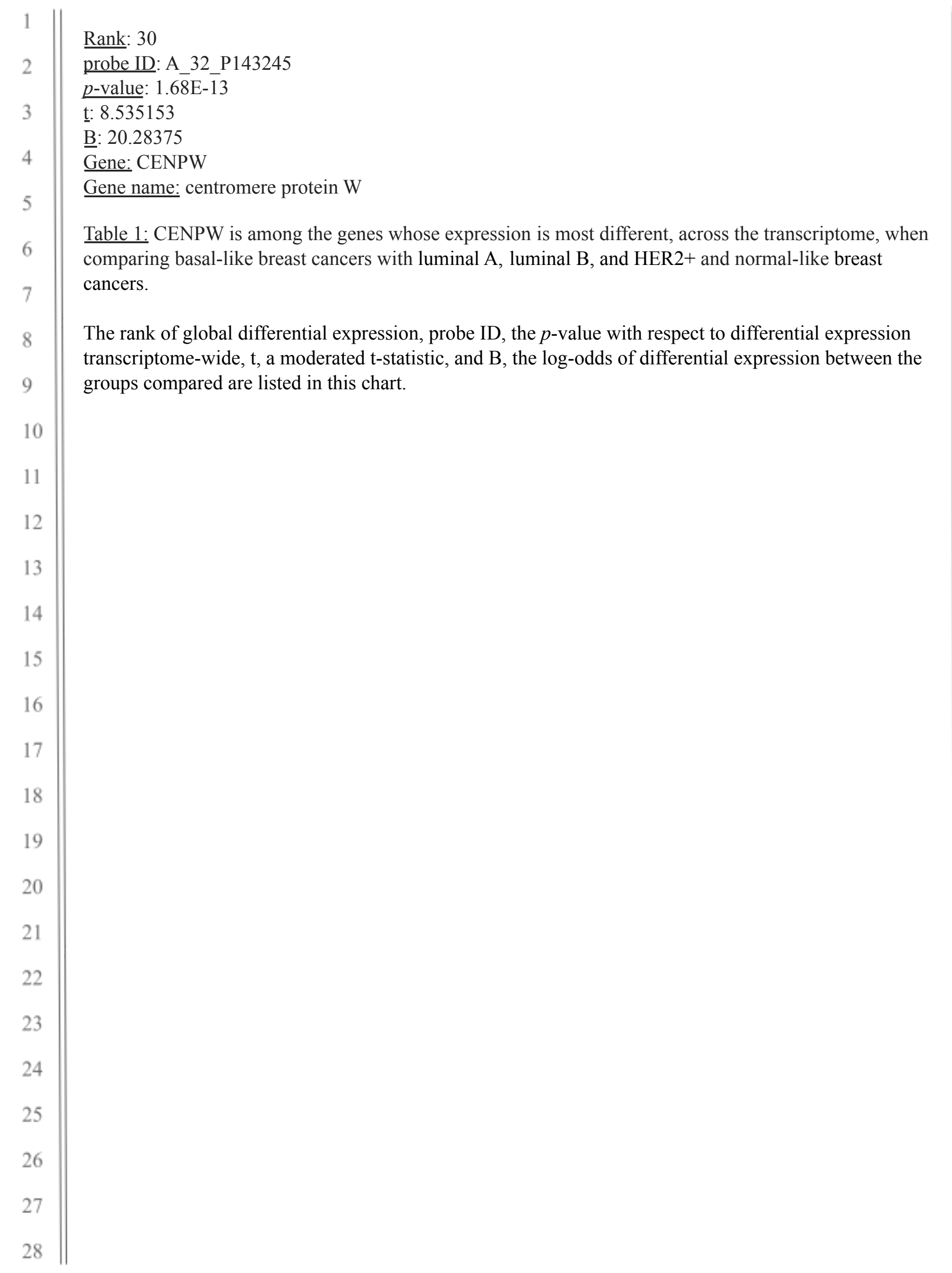




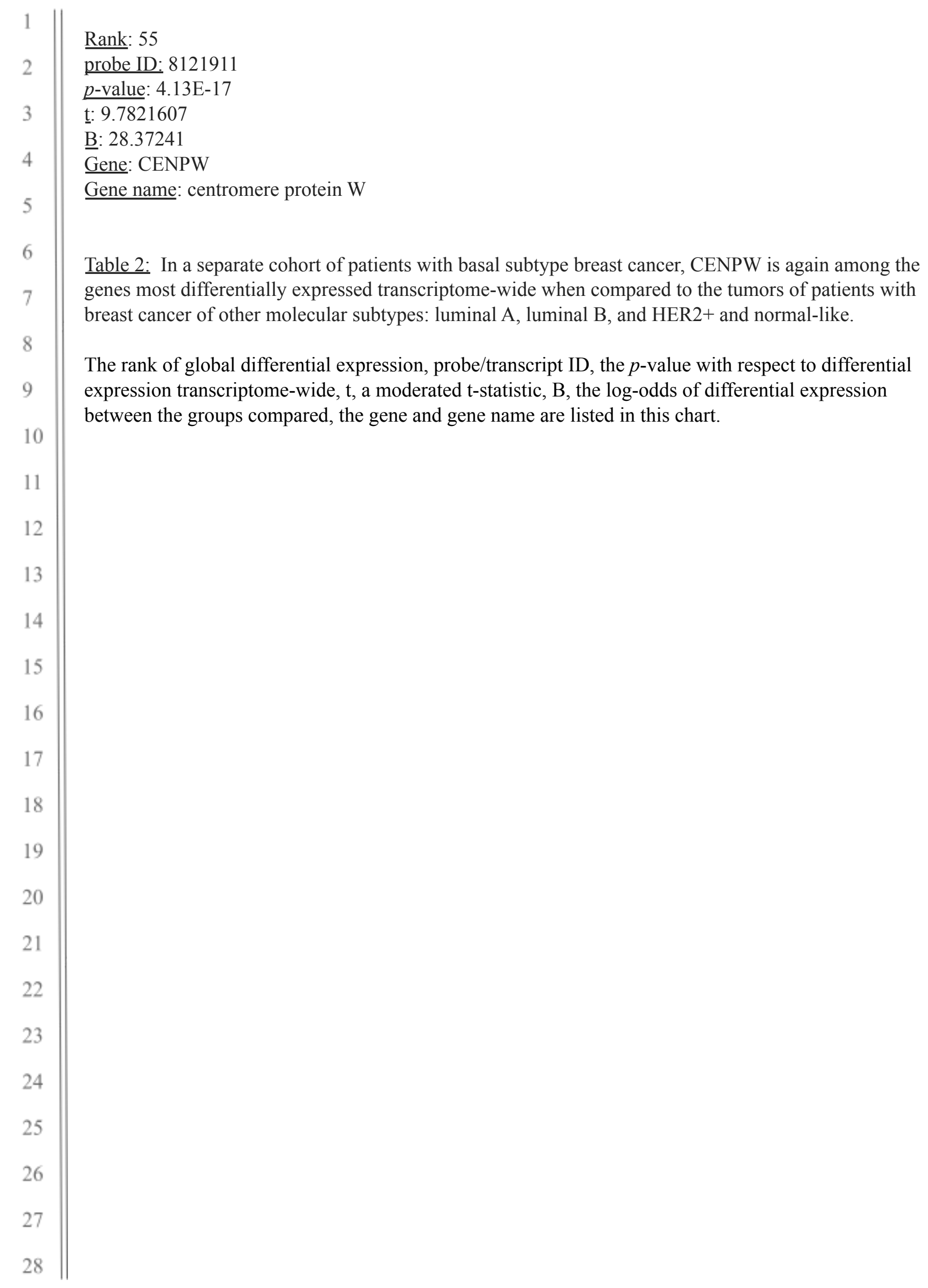




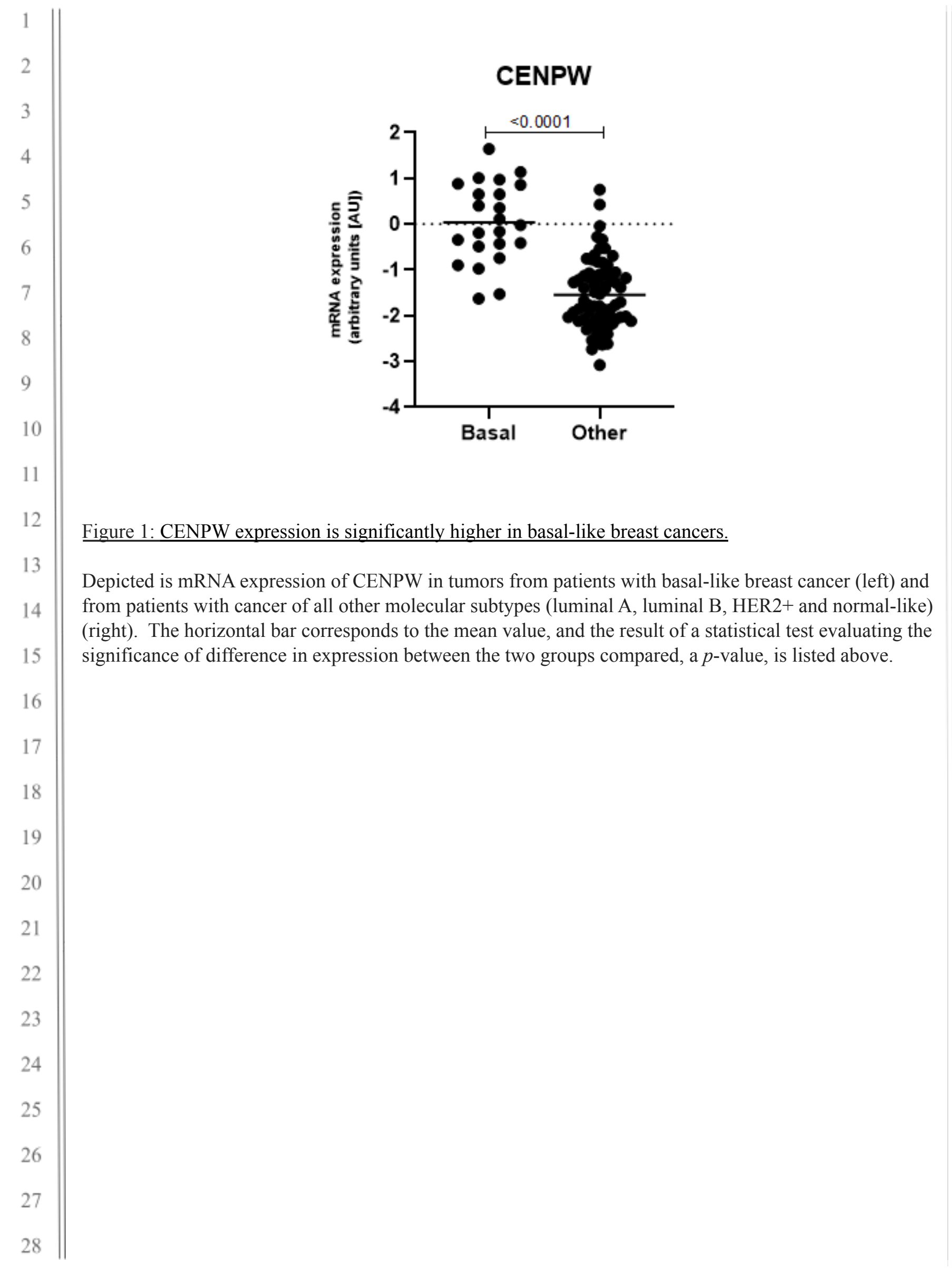




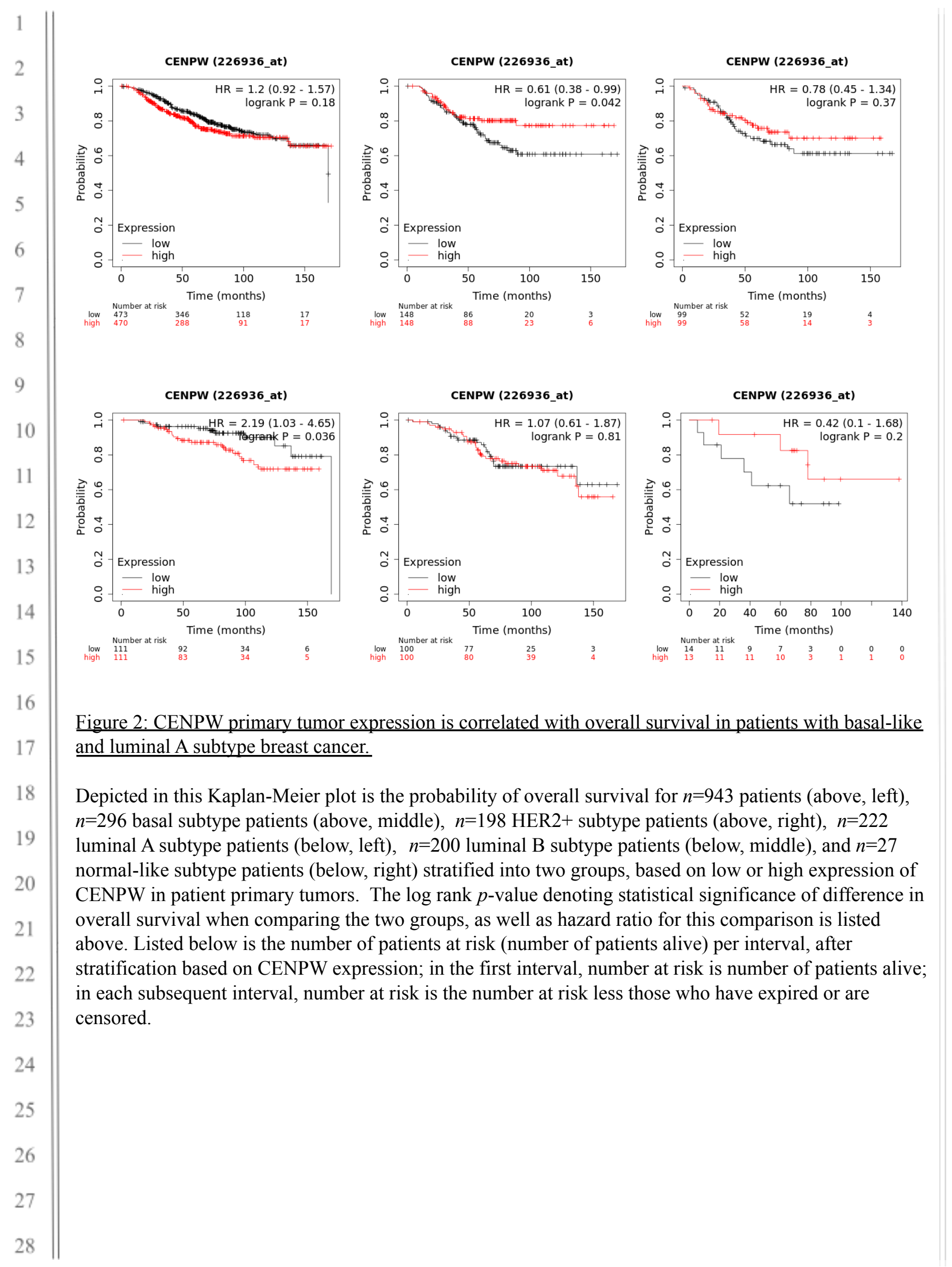




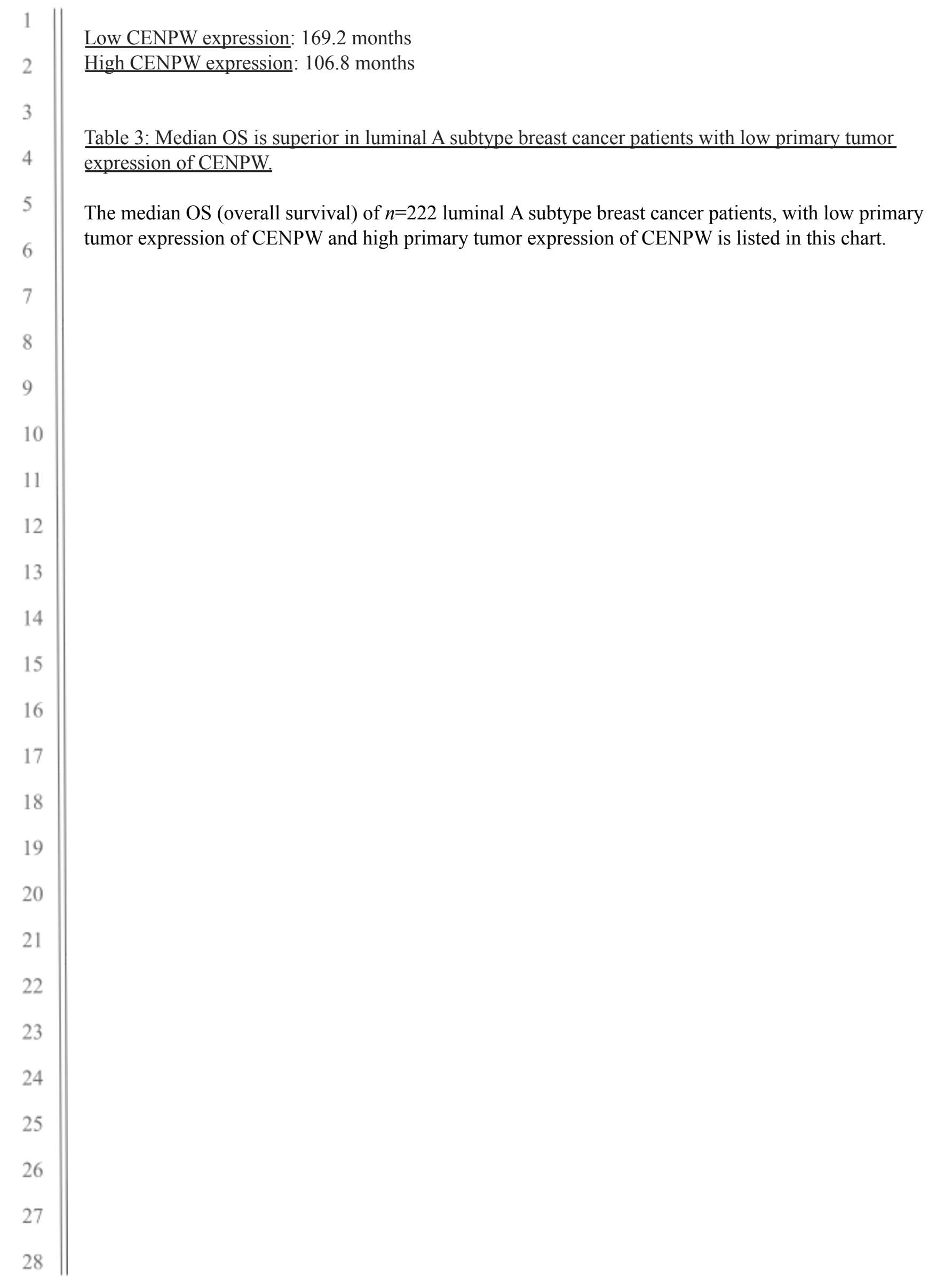

